\title{
Title: How Well Do European Patients Understand Cancer-Associated Thrombosis? A Patient Survey
}

Authors: Ismail Elalamy, Anna Falanga, Charis Girvalaki, Manuel Monreal, Jacob C Easaw, Annie Young

Ismail Elalamy

Head of Department, Hematology and Thrombosis Center, Tenon University Hospital

Département Médico-Universitaire de Biologie et Génomique Médicales BioGeM

Médecine Sorbonne Université, Paris, France

Anna Falanga

Chief Department of Immunohematology and Transfusion Medicine, Thrombosis and Hemostasis Center, Hospital Papa Giovanni XXIII, Bergamo, Italy

Charis Girvalaki

EUA Affairs Manager, European Cancer Patient Coalition, Brussels

Manuel Monreal

Professor Vascular Medicine at Faculty of Medicine, Universidad Autonoma de Barcelona, Head Internal

Medicine Hospital Universitari Germans Trias, Barcelona, Spain

Jacob C. Easaw

Oncologist/Hematologist, Department of Medical Oncology, Cross Cancer Institute, Edmonton, Alberta, Canada

Annie Young

Emerita Professor of Nursing, Warwick Clinical Trials Unit, Warwick Medical School, University of Warwick Coventry, UK

\section{SIMPLE SUMMARY}

Patients with cancer are living longer with an increased risk for cancer- and treatment-related side-effects, including an increased risk for cancer-associated thrombosis (CAT). The aim of this pan-European patient survey was to assess patient awareness and knowledge about CAT, including risk factors, signs and symptoms and interventions, to better prevent and treat CAT. Highlighting key findings from the survey, we outline the gaps and needs regarding who, when, and how information should be provided, as well as what type of information should be provided, to cancer patients and their caregivers to reduce the risk of CAT. It is important that the entire oncology multidisciplinary team participate in educating patients about CAT to help them participate in decision-making process and to improve patient outcomes. Education is best provided in a variety of approaches including a healthcare professional-patient encounter, printed material and digital/electronic/web based material. Speaking about CAT between patient, caregiver and healthcare professional along the whole continuum of cancer care provides a good model for prevention and treatment of CAT.

\begin{abstract}
Ongoing concerns regarding the morbidity and mortality from cancer-associated thrombosis led the European Cancer Patient Coalition (ECPC), the voice of cancer patients across Europe, to create a panEuropean cancer-associated awareness patient survey to assess CAT knowledge among a large population of
\end{abstract}


patients with cancer. The ECPC survey represents the largest of its kind amongst patients/caregivers with CAT and identified significant gaps in patient awareness and knowledge of CAT. It also identified a need for educational CAT-related discussions and interventions between healthcare professionals and patients with cancer and their caregivers. The aim of this paper is to highlight these gaps and to provide healthcare professionals with awareness of what information should be shared with patients/caregivers as well as how and when that information should be provided. Notably, the importance of providing information on CAT risk and risk factors, how to reduce their risk of CAT, the role of anticoagulant prophylaxis and treatment (short- and long-term) including possible side-effects, and finally how to early identify CAT symptoms. Here we outline what type of information should be provided, as well as when and how to best discuss CAT with our oncology patients and their caregivers along the cancer care continuum, to reduce the risk of CAT and associated complications with a goal of improving patient outcomes.

\section{Keywords:}

Cancer-associated thrombosis, survey, anticoagulant, cancer patients

\section{Introduction}

The introduction of new treatments has contributed to improved overall survival for cancer patients. Many patients are living longer with their cancer and remain on systemic anticancer therapies longer (approximately two times longer), resulting in an increased risk for treatment-related long-term and latent side-effects, including blood clots[1]. Despite the fact that both cancer and its various treatments are well recognized risk factors for venous thromboembolism (VTE), thrombosis remains a major cause of morbidity and mortality in this population[2,3]. Data support that up to $20 \%$ of patients with cancer will experience VTE, which is approximately 4-5 times higher than the general population[4]. Unfortunately, despite the large body of evidence demonstrating the safety and efficacy of anticoagulants in the prevention and treatment of venous thrombosis in this population [5,6], it is well recognized that patients with cancer associated thrombosis (CAT) continue to have higher hospitalization rates with increased risk of mortality and decreased rates of thrombolysis than in the general population[2,7,8]. The high rate of CAT suggests that, despite the clear guideline recommendations for patients with cancer (ISTH, ASCO, ESMO, ASH), CAT prevention and recognition remain low amongst healthcare professionals [3,9,10]. Further, several small patient surveys conducted between 2010 and 2014 have revealed that patients and their families were unaware of VTE as a complication of cancer and its treatment, expressing a desire to be educated about VTE symptoms, risk factors, prevention strategies and complications to better equip themselves in their cancer journey and improve their overall cancer outcomes[11,12].

A roundtable including oncology healthcare professionals, policymakers and patient advocates was convened discuss and review the evidence to address ongoing concerns of excessive CAT associated morbidity and mortality, as well as patients' desire for greater CAT awareness. These discussions demonstrated that greater knowledge about CAT was needed across the spectrum of healthcare practitioners and patients, particularly regarding primary and secondary prevention of thrombosis. Additionally, it was noted that improved communication throughout the entire cancer care pathway was required to raise awareness regarding CAT.

As a follow-up to this roundtable, the European Cancer Patient Coalition (ECPC), considered by many to be the voice of cancer patients across Europe, in collaboration with healthcare professionals and industry, created the first ever pan-European survey to assess the level of awareness of CAT risk, symptoms, treatments, and desired attributes for anticoagulation treatment among patients with cancer. This survey was 
designed to act as a baseline of CAT knowledge which could then inform and enable conversations about CAT with healthcare professionals as well as with patients with cancer. Future educational CAT-related interventions could then be compared to this baseline.

The ECPC survey was originally released at World Thrombosis Day in October 2018 (ECPC_2018_Cancer_associated_thrombosis_awareness_survey_report.pdf)

Here we highlight aspects of the survey that identify significant gaps in patient awareness and knowledge of CAT. Specifically, these data suggest that patients have a poor understanding of their own risk, symptoms of CAT, prevention, and treatment options for CAT. Healthcare professionals along the entire continuum of care are encouraged to speak directly more often with their patients to educate them about CAT[13].

\section{RESULTS}

Patient Characteristics

There were 1,365 cancer patients/survivors or caregivers who responded to the survey. Responses were received from 6 countries; Spain and the UK provided the highest proportion of responders (Germany $(n=175)$, Greece $(n=267)$, Italy $(n=246)$, Spain $(n=332), U K(n=324)$, and France* $(n=21))$. Of the responders, the majority $(76 \% ; n=966))$ were cancer patients/survivors and were female $(75 \%)$. About twothirds of patients had cancer for $>1$ year and $>90 \%$ were 45 years of age or older. Twenty four percent $(n=299)$ of survey participants had suffered from thrombosis associated with their cancer.

The majority of responders $(68 \% ; n=926)$ heard about the survey online reflecting the dissemination of the survey through various on-line mechanisms (social media, email newsletters, website posts and media)[13].

\section{Patient's awareness of CAT and CAT risk}

The survey found that $\mathbf{7 2} \%(\mathrm{n}=\mathbf{9 5 7})$ of survey participants were unaware of the higher than normal risk of developing thrombosis in cancer patients. When asked to rate their overall understanding of CAT on a scale of 1 (low) to 10 (high), the mean score was 4.1/10. In fact, over half of respondents (55\%) gave a rating of 4 or below and only $21 \%$ gave a rating of 7 or above (Figure 1)[13].

Figure 1: Survey Participant Rating of Their Understanding of CAT

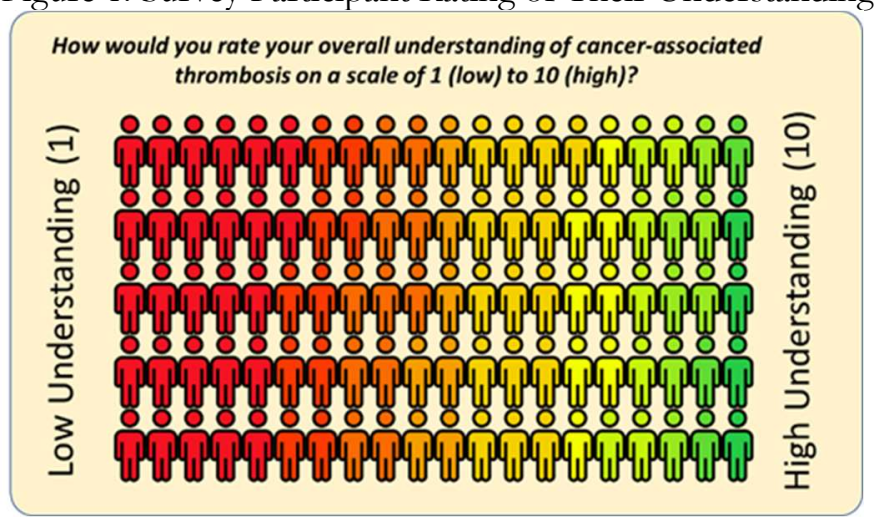

When asked about the risk factors for VTE, inactivity was the only risk factor that was recognized by more than half of the respondents. Less than half were aware of other risk factors that increase the risk of thrombosis (Table 1).

Table 1: Participant Understanding of Risk Factors for CAT

\begin{tabular}{|l|l|}
\hline Risk factor for cancer-associated thrombosis & Percentage of respondents completely aware \\
\hline
\end{tabular}




\begin{tabular}{|l|c|}
\hline Inactivity for long periods of time* & $65 \%$ \\
\hline Previous thromboses & $46 \%$ \\
\hline Cancer surgery & $35 \%$ \\
\hline Chemotherapy & $33 \%$ \\
\hline Central venous catheter (central line) & $23 \%$ \\
\hline Radiotherapy & $17 \%$ \\
\hline Certain cancer types & $15 \%$ \\
\hline
\end{tabular}

*in bed $\geq 4$ days; ${ }^{\dagger}$ certain cancer types including cancers of the stomach, brain, kidneys, ovaries

First information about $C A T$

Survey participants responded that they had received information on CAT at a variety of time points through their cancer journey, with 35\% of patients being made aware either immediately before or at their cancer diagnosis. Of particular concern, one quarter $(26 \%)$ of respondents (the largest proportion) noted that they first became aware of cancer-associated thrombosis when they suffered a blood clot (Figure 2)[13].

\section{Figure 2: First Awareness of CAT}

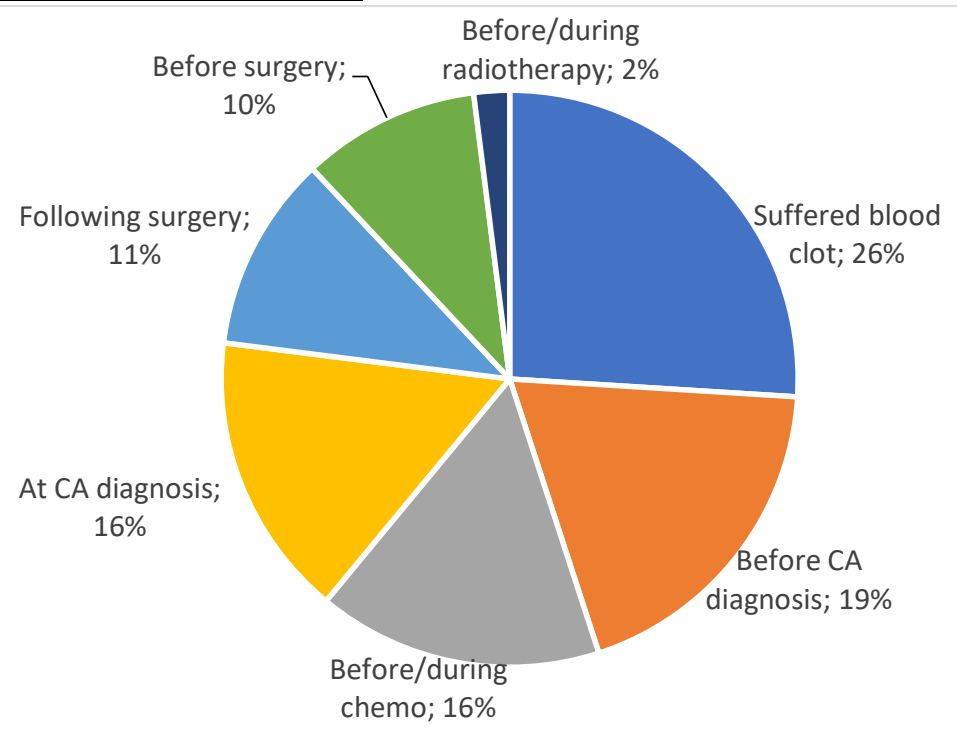

CAT Prevention and Symptoms

Amongst those completing the survey, awareness of actions they could take to reduce the risk of thrombosis varied a substantially with $87 \%(\mathrm{n}=325)$ of respondents indicating they were aware that taking a walk could reduce their risk (Figure 4)[13]. 
Figure 3: Aware that Action Could Reduce Risk of CAT

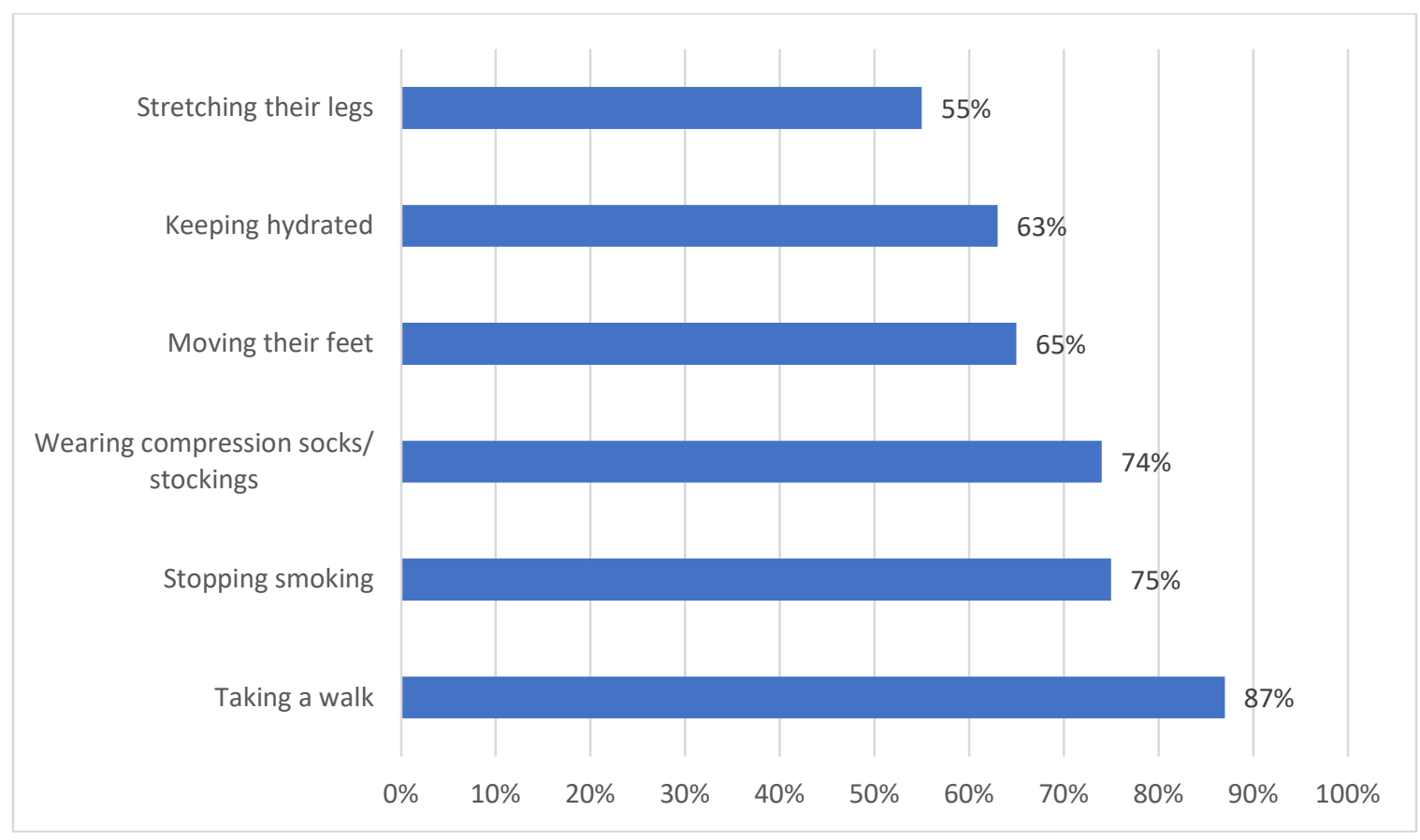

Symptoms of CAT appeared to be relatively well known, with $73 \%(n=270)$ of survey participants indicating that they were aware that swelling in the foot, ankle or leg could be a sign of deep vein thrombosis (DVT) and $71 \%$ indicating that shortness of breath could be a sign of pulmonary embolism (PE) (Figure 4). Other symptoms, however, were less well known with just over half (57\%) of participants being aware that pain, cramping, tenderness could be a sign of DVT and one third (33\%) knew that irregular heartbeat could be a sign of PE[13].

Figure 4: Understanding of DVT/PE Symptoms and What to Look For

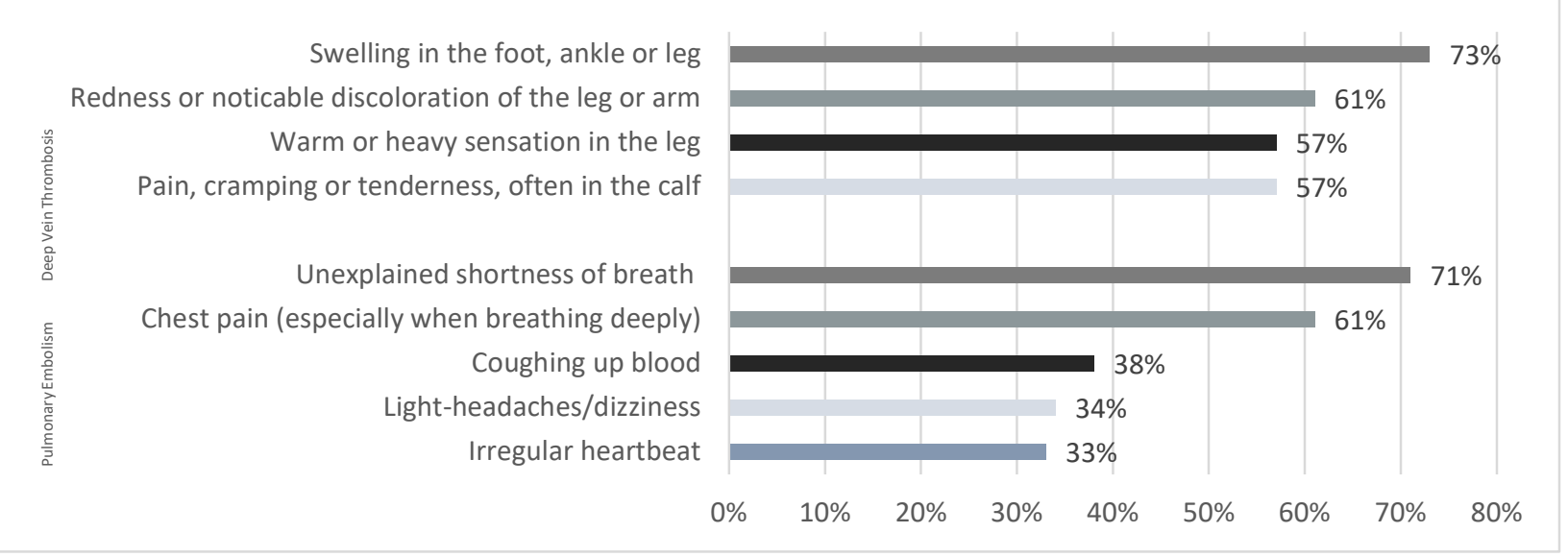

\section{Treating CAT}

Over a third of respondents (37\%) stated that they were currently using anticoagulants. Within this group, $60 \%$ were being treated for an existing clot and the remainder as VTE prophylaxis. It was noted that an equal number used an oral vs an injectable anticoagulant. Virtually all $(96 \% ; n=371) \mathrm{knew}$ that anticoagulants could be used to effectively treat thrombosis. However, only $41 \%(\mathrm{n}=136)$ had been informed about possible sideeffects including increased bleeding risk. For those patients taking an anticoagulant, $22 \%$ had experienced 
side-effects from their anticoagulants and $9 \%$ had not been informed about what to do if side-effects occurred[13].

\section{DISCUSSION}

Prior to the ECPC survey, little was known about European cancer patient awareness of thrombosis. The first key finding was that the vast majority of respondents reported that they were completely unaware that cancer patients have a higher-than-normal risk of developing cancer-related thrombosis. In fact, only $21 \%$ of patients indicated that they had a good understanding of CAT (rating of 7 or higher) supporting the need for improved CAT-related education throughout their cancer journey. The second key finding was that up to two-thirds of patients with cancer were not informed about their CAT risk nor educated by a healthcare professional on risk factors or signs and symptoms of CAT.

The data uncovered in this survey are not surprising considering previous surveys in both hospitalized patients and outpatients diagnosed with cancer-associated thromboses. These reports confirm that patients neither receive adequate teaching regarding thrombosis nor the potential harm associated with thromboses. In each study, most preferred to receive education in the context of a doctor/healthcare professional-patient encounter[11,12].

More specifically, the data in this survey suggest that information given regarding VTE risk, signs and symptoms, and treatment as well as treatment-related side effects must be provided at regular intervals by the entire oncology care team which includes physicians, nurses, and pharmacists. In addition, contact with patients during in-person and virtual clinical encounters, self-learning (e.g., internet resources and printed leaflets) should also be encouraged. This can be done through a local CAT pathway along the continuum of care (e.g., pre-surgery and chemotherapy encounters). There also exists a significant amount of online education material and patients should be given guidance on which internet sites offer the most reputable information[14].

As previously uncovered in the PELICAN studies[12,15,16] patients' experiences are an education in themselves, particularly for the oncology care team. Once the patient has a thrombosis, it is too late to act on prevention which is the most crucial focus. Through this survey we have learned about patients' with cancer awareness and knowledge of CAT and their experiences with CAT prevention and treatment. Oncology professionals, as well as other members of the patient's care team (e.g. internists, surgeons, nurses), need to perform better, at every stage of the patient's cancer pathway, to ensure patients are aware of CAT and their individual risk to develop a blood clot.

Importantly, there needs to be clear communication between oncology professionals regarding who will provide the education as well as when, and how often that education should be performed. One successful model for CAT guideline implementation was the Venous Thromboembolism Prevention in the Ambulatory Cancer Clinic (VTE-PACC) program which incorporated a multidisciplinary team of oncologists, hematologists, pharmacists, and nurses, and an electronic health record-based risk assessment that was nursing driven. This care model used a multidisciplinary approach to focus on VTE risk assessment and prophylaxis in oncology patients leveraging multiple areas of expertise to ensure patient at high risk for developing a VTE were identified and educated about their risk and risk reduction options with a focus on optimizing clinical outcomes for each patient. This model led to an increase in VTE education and risk assessment from $<5 \%$ to $>95 \%$ of outpatients with cancer. Further, $94 \%$ of high-risk patients received additional evaluation and recommendations for thromboprophylaxis. Successful implementation of VTE guidelines using a model such this may significantly impact patient morbidity and mortality[17]. Data also support that the multi-disciplinary team approach ensures that patients are provided with the education and information to help them share in the decision-making process and with improved patient engagement comes improved patient satisfaction and outcomes[18]. 
Examples of Excellent CAT Patient Material vetted for European Patients:

- $\quad$ ECPC CAT-Be clot conscious

- Anticoagulation UK CAT

CAT Education Tips

- In clinic, listening and teaching moment, followed by recommendations for websites to enable selflearning

- Enabling multidisciplinary team, including community pharmacists, to provide VTE disease education (ClotAssist program)[19]

Appropriate information about CAT risk should include lesser known but equally important factors, including cancer surgery, the use of central lines, and the increased CAT risks associated with specific cancers, chemotherapeutics, and radiotherapy. Patients also need more information about actions they can take to reduce their risk of CAT, particularly those that are relatively easy and low cost to implement such as walking if possible and keeping hydrated. Further, patients should also be fully informed about symptoms of CAT and who to contact if they experience symptoms such as warmth, pain, swelling tenderness or redness in any extremity, particularly the calf or leg. For pulmonary embolism, symptom awareness should include chest discomfort, shortness of breath coughing blood, light-headedness/dizziness, and an irregular heartbeat[3].

Specific limitations with this survey have been identified. First, this was a self-reported assessment of cancer diagnosis and CAT awareness and, being voluntary, it is likely that those who participated had a particular interest in VTE, possibly because of their experience as a patient or caregiver. Further, as the survey was only available to those in the UK and certain European countries, differences in the healthcare systems between countries may also impact the outcome. The survey was primarily completed online (only 30 paper copies were completed), favouring patients with access to a computer, suggesting affordability and health status. As well, not all questions were answered by all respondents, resulting in missing data which could potentially have impacted analysis, and finally there is a lack of comparator population. However, similarities between the answers from participants across multiple countries lends credibility to the understanding and development of consistent themes[13].

\section{MATERIALS AND METHODS}

The cancer-associated awareness patient survey was initiated by the ECPC in conjunction with other relevant member organizations to identify themes and develop questions to better understand awareness of CAT. The survey, consisting of 35 questions, was conducted by Quality Health on behalf of the ECPC between April and Sept 2018 in 6 countries (UK, France, Germany, Greece, Italy and Spain). The survey questions included contextual questions (demographic information/status of treatment) as well as questions aligned along treatment pathways (diagnosis, testing, status of treatment) regarding from whom and how information on CAT was acquired and could be answered by cancer patients/survivors or their caregivers. The questions provided predefined answer options based on best principles for surveys to ensure optimal responses. The survey was set up on Quality Health's bespoke on-line survey system, with the survey and accompanying introduction information translated to French, German, Greek, Italian and Spanish. Dissemination of the survey was carried out through ECPC's membership network using social media, email newsletters, website posts, and through media work[13].

Data analysis: A three-stage process was undertaken in the development and analysis of the survey including development and beta-testing of the questions and platform, patient completion in 6 EU countries, followed by a thorough analysis and reporting of the findings with an overall summary (combined data from all 
respondents in all countries) as well as an analysis by country. Percentages are calculated after excluding those participants that did not answer that particular question. All percentages are rounded to the nearest whole number. When added together, the percentages for all answers to a particular question may not total $100 \%$ because of this rounding. Data is included in the overall findings; a separate analysis of the French findings was not completed as numbers would not allow for robust statistical analysis[13].

\section{CONCLUSION}

It is evident from this large survey of cancer patient/survivors that oncology professionals need to better understand the gaps in patient knowledge regarding cancer-associated thrombosis. Further, healthcare professionals need to provide education to patients across the continuum of care to improve patient outcomes. We have a collective responsibility using the ECPC patient survey as a baseline to inform patients with cancer on how to identify signs and symptoms of CAT to enable faster diagnosis and treatment. Improved education regarding the benefits and risks of using anticoagulant therapy also needs to be emphasized. We have provided an example of a successful patient education model implemented throughout the continuum of care that could be adapted and implemented in cancer centers across Europe. We have also provided excellent evidence-based online resources that be readily accessed by healthcare professionals.

Ultimately, patient outcomes will be improved if we recognize and develop cogent strategies to educate our patients on the risk factors, treatment and treatment-related side effects associated with blood clots in cancer patients.

\section{Back Matter}

Funding: The development of this manuscript and publication costs were supported by a grant from LEO Pharma Inc.

Acknowledgements: The authors acknowledge writing and editorial support by Bonnie Kuehl, $\mathrm{PhD}$ in the development of the manuscript.

Author Contribution: Each author contributed equally in the interpretation of the data and creation of the manuscript.

The authors declare no conflict of interest.

The sponsors had no role in the design, execution, interpretation, or writing of the manuscript.

\section{References}

1. Gegechkori, N.; Haines, L.; Lin, J.J. Long-Term and Latent Side Effects of Specific Cancer Types. Med Clin North Am 2017, 101, 1053-1073, doi:10.1016/j.mcna.2017.06.003.

2. Khorana, A.A.; Francis, C.W.; Culakova, E.; Kuderer, N.M.; Lyman, G.H. Thromboembolism Is a Leading Cause of Death in Cancer Patients Receiving Outpatient Chemotherapy. J Thromb Haemost 2007, 5, 632-634, doi:10.1111/j.1538-7836.2007.02374.x.

3. Key, N.S.; Khorana, A.A.; Kuderer, N.M.; Bohlke, K.; Lee, A.Y.Y.; Arcelus, J.I.; Wong, S.L.; Balaban, E.P.; Flowers, C.R.; Francis, C.W.; et al. Venous Thromboembolism Prophylaxis and Treatment in Patients With Cancer: ASCO Clinical Practice Guideline Update. J Clin Oncol 2020, 38, 496-520, doi:10.1200/JCO.19.01461.

4. Trujillo-Santos, J.; Martos, F.M.; Font, C.; Farge-Bancel, D.; Rosa, V.; Lorenzo, A.; Barrón, M.; Lorente, M.A.; Pedrajas, J.M.; Monreal, M. Analysis of Clinical Factors Affecting the Rates of Fatal Pulmonary 
Embolism and Bleeding in Cancer Patients with Venous Thromboembolism. Heliyon 2017, 3, e00229, doi:10.1016/j.heliyon.2016.e00229.

5. Carrier, M.; Cameron, C.; Delluc, A.; Castellucci, L.; Khorana, A.A.; Lee, A.Y.Y. Efficacy and Safety of Anticoagulant Therapy for the Treatment of Acute Cancer-Associated Thrombosis: A Systematic Review and Meta-Analysis. Thromb Res 2014, 134, 1214-1219, doi:10.1016/j.thromres.2014.09.039.

6. Li, A.; Garcia, D.A.; Lyman, G.H.; Carrier, M. Direct Oral Anticoagulant (DOAC) versus LowMolecular-Weight Heparin (LMWH) for Treatment of Cancer Associated Thrombosis (CAT): A Systematic Review and Meta-Analysis. Thromb Res 2019, 173, 158-163, doi:10.1016/j.thromres.2018.02.144.

7. Haas, S.; Ageno, W.; Weitz, J.I.; Goldhaber, S.Z.; Turpie, A.G.G.; Goto, S.; Angchaisuksiri, P.; Nielsen, J.D.; Kayani, G.; Zaghdoun, A.; et al. Anticoagulation Therapy Patterns for Acute Treatment of Venous Thromboembolism in GARFIELD-VTE Patients. Journal of Thrombosis and Haemostasis 2019, 17, 1694 1706, doi:https://doi.org/10.1111/jth.14548.

8. $\quad$ Ba Aqeel, S.H.; Lingamaneni, P.; Farooq, M.Z.; Baral, B.; Banskota, S.U.; Gupta, S. Nationwide Analysis of Healthcare Burden and Risk of Mortality in Cancer Patients with Deep Venous Thrombosis. JCO 2020, 38, e19093-e19093, doi:10.1200/JCO.2020.38.15_suppl.e19093.

9. Lyman GH, Carrier M, Ay C, et al. American Society of Hematology 2021 Guidelines for Management of Venous Thromboembolism: Prevention and Treatment in Patients with Cancer. Blood Adv. 2021;5(4):927-974. Blood Adv 2021, 5, 1953, doi:10.1182/bloodadvances.2021004734.

10. Farge, D.; Frere, C.; Connors, J.M.; Ay, C.; Khorana, A.A.; Munoz, A.; Brenner, B.; Kakkar, A.; Rafii, H.; Solymoss, S.; et al. 2019 International Clinical Practice Guidelines for the Treatment and Prophylaxis of Venous Thromboembolism in Patients with Cancer. Lancet Oncol 2019, 20, e566-e581, doi:10.1016/S1470-2045(19)30336-5.

11. Popoola, V.O.; Lau, B.D.; Shihab, H.M.; Farrow, N.E.; Shaffer, D.L.; Hobson, D.B.; Kulik, S.V.; Zaruba, P.D.; Shermock, K.M.; Kraus, P.S.; et al. Patient Preferences for Receiving Education on Venous Thromboembolism Prevention - A Survey of Stakeholder Organizations. PLoS One 2016, 11, e0152084, doi:10.1371/journal.pone.0152084.

12. Noble, S.; Prout, H.; Nelson, A. Patients' Experiences of LIving with CANcer-Associated Thrombosis: The PELICAN Study. Patient Prefer Adherence 2015, 9, 337-345, doi:10.2147/PPA.S79373.

13. European Cancer Patient Coalition. Cancer-Associated Thrombosis Awareness Survey Results Report. October 2018.

14. National Institutes of Health, National Institute of Aging Online Health Information: Is It Reliable? Available online: http://www.nia.nih.gov/health/online-health-information-it-reliable (accessed on 29 May 2021).

15. Font, C.; Nelson, A.; Garcia-Fernandez, T.; Prout, H.; Gee, P.; Noble, S. Patients' Experience of Living with Cancer-Associated Thrombosis in Spain (PELICANOS). Support Care Cancer 2018, 26, 3233-3239, doi:10.1007/s00520-018-4174-5.

16. Mahé, I.; Chidiac, J.; Pinson, M.; Pinson, M.; Swarnkar, P.; Nelson, A.; Noble, S. Patients Experience of Living with Cancer Associated Thrombosis in France (Le PELICAN). Thromb Res 2020, 194, 66-71, doi:10.1016/j.thromres.2020.06.024.

17. Holmes, C.E.; Ades, S.; Gilchrist, S.; Douce, D.; Libby, K.; Rogala, B.; Parenteau, E.; Cushman, M.; Holm, A.K. Successful Model for Guideline Implementation to Prevent Cancer-Associated Thrombosis: Venous Thromboembolism Prevention in the Ambulatory Cancer Clinic. JCO Oncol Pract 2020, 16, e868-e874, doi:10.1200/JOP.19.00697.

18. Selby, P.; Popescu, R.; Lawler, M.; Butcher, H.; Costa, A. The Value and Future Developments of Multidisciplinary Team Cancer Care. Am Soc Clin Oncol Educ Book. 2019, 39, 332-340, doi:10.1200/EDBK_236857.

19. Easaw, J.C.; McCall, S.; Azim, A. ClotAssist: A Program to Treat Cancer-Associated Thrombosis in an Outpatient Pharmacy Setting. J Oncol Pharm Pract 2019, 25, 818-823, doi:10.1177/1078155218760704. 